\title{
A!
}

This is an electronic reprint of the original article.

This reprint may differ from the original in pagination and typographic detail.

Solinas, Paolo; Möttonen, Mikko; Salmilehto, J.; Pekola, Jukka P.

\section{Cooper-pair current in the presence of flux noise}

Published in:

Physical Review B

DOI:

10.1103/PhysRevB.85.024527

Published: 20/01/2012

Document Version

Publisher's PDF, also known as Version of record

Please cite the original version:

Solinas, P., Möttonen, M., Salmilehto, J., \& Pekola, J. P. (2012). Cooper-pair current in the presence of flux noise. Physical Review B, 85(2), 1-9. [024527]. https://doi.org/10.1103/PhysRevB.85.024527

This material is protected by copyright and other intellectual property rights, and duplication or sale of all or part of any of the repository collections is not permitted, except that material may be duplicated by you for your research use or educational purposes in electronic or print form. You must obtain permission for any other use. Electronic or print copies may not be offered, whether for sale or otherwise to anyone who is not an authorised user. 


\title{
Cooper-pair current in the presence of flux noise
}

\author{
P. Solinas, ${ }^{1,2, *}$ M. Möttönen, ${ }^{1,2}$ J. Salmilehto, ${ }^{1}$ and J. P. Pekola ${ }^{2}$ \\ ${ }^{1}$ Department of Applied Physics/COMP, Aalto University, P.O. Box 14100, FI-00076 Aalto, Finland \\ ${ }^{2}$ Low Temperature Laboratory, Aalto University, P.O. Box 13500, FI-00076 Aalto, Finland
}

(Received 26 October 2011; revised manuscript received 21 December 2011; published 20 January 2012)

\begin{abstract}
We study the effect of flux noise on the Cooper pair current of a superconducting charge pump. We generalize the definition of the current in order to take into account the contribution induced by the environment. It turns out that this dissipative current vanishes for charge noise but it is finite in general for noise operators that do not commute with the charge operator. We discuss in a generic framework the effect of flux noise and present a way to engineer it by coupling the system to an additional external circuit. We calculate numerically the pumped charge through the device by solving the master equation for the reduced density matrix of the system and show how it depends on the coupling to the artificial environment.
\end{abstract}

DOI: 10.1103/PhysRevB.85.024527

PACS number(s): 03.65.Yz, 85.25.Cp, 85.25.Dq

\section{INTRODUCTION}

Geometric phases are ubiquitous in quantum physics. Both the Abelian Berry phase ${ }^{1}$ and the non-Abelian ${ }^{2}$ generalization were theoretically discovered in the eighties. During the last decade, there has been renewed interest in their possible use as tools to manipulate quantum information. The so-called geometric ${ }^{3}$ or holonomic quantum computation ${ }^{4}$ relies on the fact that it is possible to build unitary transformations which depend only on geometric properties of an abstract parameter space. The main advantage of this approach is that such geometric transformations are robust against certain types of noise. $^{5,6}$

While the Berry phase has been observed in many quantum systems, ${ }^{7-9}$ clear experimental evidence of the non-Abelian adiabatic geometric phases is still missing despite different proposals for their theoretical implementation. ${ }^{10-16}$ One of the main reasons for this is that the system must be cyclically steered in an adiabatic fashion. In other words, the time to implement a geometric non-Abelian transformation is long with respect to the dynamical time scale of the system. This renders it difficult to protect the system from environmental noise and thus decoherence can become an issue in the implementation of geometric quantum computation.

A step toward understanding the effect of the environment and the robustness of steered quantum evolution was taken in Refs. 17-19, where it has been shown that ground-state evolution is robust against relaxation and dephasing induced by a low-temperature dissipative element.

Among different physical systems, the Cooper pair sluice ${ }^{20}$ is a promising candidate for studying environmental effects on geometric phases. In fact, the pumped charge through the Cooper-pair sluice is known to be directly connected to the geometric phases. ${ }^{21-24}$ This has allowed measurement of the Berry phase in such a system. ${ }^{9}$ This has paved the way for ground-state geometric quantum computating in which the quantum information is manipulated through a geometric operator but the system is kept in a doubly degenerate ground state during the whole evolution..$^{24,25}$

Furthermore, transmon quibits coupled to a superconducting cavity offer potential systems for the observation of the non-Abelian phases. ${ }^{26}$ They constitute the other superconducting platform in which the Berry phase has been observed experimentally. $8,27,28$

The Cooper-pair sluice operates in the charging regime; i.e., the charging energy dominates over the Josephson energy. For this reason, the noise induced by charge fluctuations is likely to be dominant and it has been studied in Refs 17 and 18. In this paper, we analyze the effect of another kind of noise: flux noise. This can be induced, for example, by a fluctuating magnetic field. We find that the definition of the current must be extended to include the contribution induced by the environment. This dissipative current is directly related to the symmetry of the system and can be calculated using the master equation approach.

As a practical application, we discuss the effect of flux noise on Cooper pair pumping. Instead of a purely theoretical approach, we study a possible implementation in a realistic experimental setup. We couple the Cooper-pair sluice to an artificial noise source produced by an external circuit. The main advantage of this approach is the possibility of controlling in situ the coupling strength between the system and the noise source.

The article is organized as follows. In Sec. II, we derive the general expression for the current operator, with the emphasis initially on the symmetries of the problem and then on the connection with the master equation. In Sec. III, we apply these results to the pumping process and identify the different contributions to the current and the pumped charge due to the environment. We analyze the particular case of flux noise which perturbs the phase across the superconducting loop. Section IV presents the circuits for engineering the artificial flux noise environment. In Sec.V, we discuss how the pumped charge is influenced by artificial noise.

\section{EVOLUTION OF OPERATOR EXPECTATION VALUES}

Let us assume that a quantum system is in a state described by the density matrix $\hat{\rho}$. The expectation value of a quantum observable $\hat{A}$ is

$$
\langle\hat{A}\rangle=\operatorname{Tr}(\hat{\rho} \hat{A})=\sum_{i}\langle i|\hat{\rho} \hat{A}| i\rangle,
$$

where the last term is the explicit expression in a timeindependent basis $\{|i\rangle\}$. If $\hat{A}$ is time independent, the time 
derivative of the expectation value is

$$
\frac{d}{d t}\langle\hat{A}\rangle=\sum_{i}\left\langle i\left|\frac{d \hat{\rho}}{d t} \hat{A}\right| i\right\rangle=\operatorname{Tr}\left(\frac{d \hat{\rho}}{d t} \hat{A}\right) .
$$

Using the von Neumann equation we obtain the well-known Ehrenfest theorem

$$
\frac{d}{d t}\langle\hat{A}\rangle=-\frac{i}{\hbar} \operatorname{Tr}(\hat{\rho}[\hat{A}, \hat{H}]),
$$

where $\hat{H}$ is the Hamiltonian which determines the dynamics. The above equation allows the definition of the current associated with the $\hat{A}$ operator, that is, it describes the respective temporal change. The definition reduces to the case of the electric current if $\hat{A}$ is selected as the charge operator.

The obtained result is valid for a general quantum system. Let us consider the case in which the total system is composed of the subsystem $S$ and the environment $E$. We are interested in the dynamics and observables related to the subsystem $S$. To simplify the discussion, in the following, we refer to $S$ simply as the system. The total Hamiltonian can be written as $\hat{H}=\hat{H}_{S}+\hat{H}_{E}+\hat{H}_{I}$, where $\hat{H}_{S}$ denotes the Hamiltonian of the system, $\hat{H}_{E}$ is the Hamiltonian of the environment, and $\hat{H}_{I}$ describes the interaction between the system and the environment. The $\hat{A}$ operator acts only on the system space, i.e., $\left[\hat{H}_{E}, \hat{A}\right]=0$, and from Eq. (3) we obtain

$$
\frac{d}{d t}\langle\hat{A}\rangle=-\frac{i}{\hbar}\left\{\operatorname{Tr}\left(\hat{\rho}\left[\hat{A}, \hat{H}_{S}\right]\right)+\operatorname{Tr}\left(\hat{\rho}\left[\hat{A}, \hat{H}_{I}\right]\right)\right\} .
$$

The trace in Eq. (4) is over all the degrees of freedom and can be split into the trace over the degrees of freedom of the system and the environment: $\operatorname{Tr}(\hat{A})=\operatorname{Tr}_{S}\left[\operatorname{Tr}_{E}(\hat{A})\right]$. By noticing that both $\hat{A}$ and $\hat{H}_{S}$ act only on the system degrees of freedom and that $\operatorname{Tr}_{E}(\hat{\rho})=\hat{\rho}_{S}$ (where $\hat{\rho}_{S}$ is the reduced density operator of the system), we obtain

$$
\frac{d}{d t}\langle\hat{A}\rangle=-\frac{i}{\hbar}\left\{\operatorname{Tr}_{S}\left(\hat{\rho}_{S}\left[\hat{A}, \hat{H}_{S}\right]\right)+\operatorname{Tr}\left(\hat{\rho}\left[\hat{A}, \hat{H}_{I}\right]\right)\right\} .
$$

The two contributions in the current associated with $\hat{A}$ have different origins. The first term on the right-hand side of Eq. (5) resembles the current for an isolated system. In fact, if the system does not interact with the environment, $\hat{H}_{I}=0$, this is the only contribution present. The second term can be interpreted as an additional contribution to the current due to the interaction with the environment. In the following, we refer to the two contributions as the system current and the dissipative current, respectively.

The case in which $\hat{H}_{I}$ commutes with the $\hat{A}$ operator is particularly interesting. Here the dissipative contribution to the current vanishes. However, the interaction with the environment influences the evolution of $\hat{\rho}_{S}$ and thus it can modify the current.

\section{A. Connection to the master equation}

Equation (5) is written in order to emphasize the symmetry of the problem. However, if the system and the environment have no particular symmetry, it cannot be used in general to estimate analytically the contribution of the dissipative current since the calculation involves the full density operator.
The dynamics of the reduced density matrix of the system $\hat{\rho}_{S}$ is obtained by writing the formal solution of the von Neumann equation, expanding it in powers of the systemenvironment coupling, and then taking the trace with respect to the environmental degrees of freedom. ${ }^{29}$ With this procedure we arrive at a standard form of the master equation,

$$
\frac{d \hat{\rho}_{S}}{d t}=-\frac{i}{\hbar}\left[\hat{H}_{S}, \hat{\rho}_{S}\right]+\hat{\mathcal{L}},
$$

where $\hat{\mathcal{L}}$ includes all the contributions due to the environment.

We can apply this formalism to the case discussed above. As assumed in the derivation of the master equation, $\operatorname{Tr}_{E}\left(\frac{d \hat{\rho}}{d t}\right)=$ $\frac{d \hat{\rho}_{S}}{d t}$, and thus, $\operatorname{Tr}\left(\frac{d \hat{\rho}}{d t} \hat{A}\right)=\operatorname{Tr}_{S}\left\{\operatorname{Tr}_{E}\left(\frac{d \hat{\rho}}{d t} \hat{A}\right)\right\}=\operatorname{Tr}_{S}\left(\frac{d \hat{\rho}_{S}}{d t} \hat{A}\right)$. Using this result, Eqs. (2), and (6), we have

$$
\frac{d}{d t}\langle\hat{A}\rangle=-\frac{i}{\hbar} \operatorname{Tr}_{S}\left(\left[\hat{H}_{S}, \hat{\rho}_{S}\right] \hat{A}\right)+\operatorname{Tr}_{S}(\hat{\mathcal{L}} \hat{A}) .
$$

The first contribution on the right-hand side matches with the system current in Eq. (5), and hence, we have an explicit expression for the dissipative current:

$$
-\frac{i}{\hbar} \operatorname{Tr}\left(\hat{\rho}\left[\hat{A}, \hat{H}_{I}\right]\right)=\operatorname{Tr}_{S}(\hat{\mathcal{L}} \hat{A})
$$

This equation allows us to calculate the dissipative contribution to the current using the expression of $\hat{\mathcal{L}}$ from the master equation for the reduced system dynamics.

This analysis has similarities to the one presented in Refs. 30-32 in the specific case of the usual current operator associated with the charge operator. Equation (8) has important implications involving the conservation of physical quantities during dissipative evolution as discussed in Ref. 33.

\section{APPLICATION TO CHARGE PUMPING}

The above analysis helps us to set the framework for discussing the pumping process in the presence of an environment. We consider the Cooper-pair sluice ${ }^{20,34,35}$ shown in Fig. 1. It consists of a superconducting island separated by two superconducting quantum interference devices (SQUIDs) with controllable effective Josephson energies $J_{L, R}$. The electrostatic potential on the island can be controlled by varying the gate voltage $V_{g}$. The experimental access to the parameters $J_{L, R}$ and $V_{g}$ allows for full control of the quantum system and makes it an excellent prototype for different applications. Several steps have been taken in the study of the connection between Cooper-pair pumping and geometric phases, in both its Abelian ${ }^{9,22}$ and its non-Abelian version, ${ }^{23-25}$ the robustness of the ground-state pumping, ${ }^{17-19,36-38}$ and the geometric Landau-Zener-Stückelberg interferometry. ${ }^{39}$ Analogous systems have been studied for the relations between pumping and topological phases. ${ }^{40,41}$

The Hamiltonian of the sluice $\hat{H}_{S}$ is the sum of the charging Hamiltonian $\hat{H}_{\mathrm{ch}}=E_{C}\left(\hat{n}-n_{g}\right)^{2}$ and the Josephson Hamiltonian $^{34,42}$

$$
\hat{H}_{J}=-J_{L} \cos \left(\frac{\hat{\varphi}}{2}-\hat{\theta}\right)-J_{R} \cos \left(\frac{\hat{\varphi}}{2}+\hat{\theta}\right),
$$

where $\hat{\varphi}=\hat{\varphi}_{R}+\hat{\varphi}_{L}$ is the superconducting phase difference between the two leads, $n_{g}=C_{g} V_{g} /(2 e)$ is the normalized gate charge, $E_{C}=2 e^{2} / C_{\Sigma}$ is the charging energy of the sluice, $C_{g}$ 
(a)

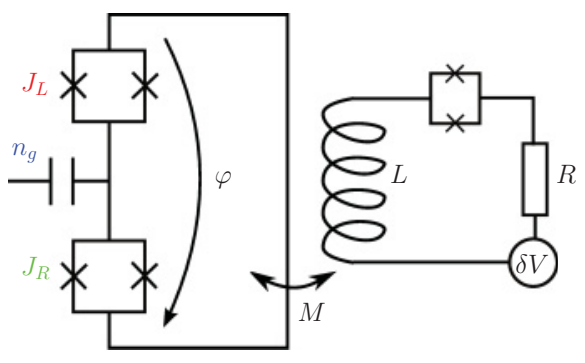

(b)

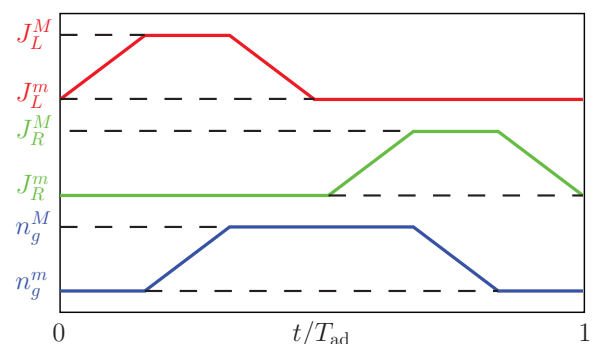

FIG. 1. (Color online) (a) Circuit diagram of the Cooper-pair sluice (left) and the artificial environment circuit (right). The experimentally controlled parameters are $J_{L, R}$ and $n_{g}, \varphi=2 \pi \Phi / \Phi_{0}$ is the phase difference across the device, and $\Phi_{0}$ is the flux quantum. The system is coupled to the artificial environment through mutual inductance $M$. The engineered environment is composed of a resistor $R$ associated with a voltage source $\delta V$, an inductor $L$, and an experimentally controllable SQUID. The SQUID allows us to change the effective noise spectrum of the environment. (b) Time dependence of the parameters $J_{L, R}$ and $n_{g}$ during a Cooper-pair pumping cycle.

is the gate capacitance, and $C_{\Sigma}$ is the total capacitance of the island. We denote by $\hat{n}_{k}=-i \partial_{\varphi_{k}}(k=L, R)$ the Cooperpair number operator of the $k$ th SQUID, and by $\hat{\theta}=\left(\hat{\varphi}_{R}-\right.$ $\left.\hat{\varphi}_{L}\right) / 2$ and $\hat{n}=-i \partial_{\theta}$ the operators for the superconducting phase and the number operator of excess Cooper pairs on the island. If the device operates in the charge regime, i.e., $E_{C} \gg \max \left\{J_{L}, J_{R}\right\}=J_{L, R}^{M}$, and the gate parameter is close to a half-integer, only the two lowest energy charge states are important for the dynamics and we can adopt the twostate approximation. Let $|0\rangle$ and $|1\rangle$ denote the states with no and one excess Cooper pair on the island, respectively. For the system under consideration, it is convenient to reduce the Hilbert space, restricting it to states with well-defined $\varphi$. In this case, $\varphi=2 \pi \Phi / \Phi_{0}$ can be treated as a real number and it is determined by the magnetic flux through the large superconducting loop $\Phi$ in Fig. 1(a).

Starting from the definition of the charge operator through the $k$ th SQUID $\hat{Q}_{k}=-2 e \hat{n}_{k}$, the discussion in Sec. II can be interpreted in terms of physical quantities. If the dynamics is influenced by the environment, the average total current through the $k$ th SQUID is

$$
\begin{aligned}
\left\langle\hat{I}_{k}^{\mathrm{tot}}\right\rangle= & \frac{d}{d t}\left\langle\hat{Q}_{k}\right\rangle=\frac{2 i e}{\hbar} \operatorname{Tr}_{S}\left(\hat{\rho}_{S}\left[\hat{n}_{k}, \hat{H}_{S}\right]\right) \\
& +\frac{2 i e}{\hbar} \operatorname{Tr}\left(\hat{\rho}\left[\hat{n}_{k}, \hat{H}_{I}\right]\right) .
\end{aligned}
$$

The current operator for a closed system is usually defined as $\hat{I}_{k}=\frac{2 i e}{\hbar}\left[\hat{n}_{k}, \hat{H}_{S}\right]$ and it corresponds to the first term on the right-hand side in Eq. (10) (see Ref. 22). Using Eq. (8), we have

$$
\left\langle\hat{I}_{k}^{\text {tot }}\right\rangle=\operatorname{Tr}_{S}\left(\hat{\rho}_{S} \hat{I}_{k}\right)+\operatorname{Tr}_{S}\left(\hat{\mathcal{L}} \hat{Q}_{k}\right)
$$

The second contributions in Eqs. (10) and (11) represent an additional dissipative current,

$$
\left\langle\hat{I}_{k}^{\mathrm{diss}}\right\rangle=\frac{2 i e}{\hbar} \operatorname{Tr}\left(\hat{\rho}\left[\hat{n}_{k}, \hat{H}_{I}\right]\right)=\operatorname{Tr}_{S}\left(\hat{\mathcal{L}} \hat{Q}_{k}\right),
$$

induced by the environment.

It is convenient to write Eq. (11) in the eigenbasis of $\hat{H}_{S}$. Let $|g\rangle$ and $|e\rangle$ be the eigenstates of $\hat{H}_{S}$ and $M_{m n}=$ $\langle m|\hat{M}| n\rangle$, with $m, n=g, e$ for any operator $\hat{M}$. Equation (11) becomes

$$
\begin{aligned}
\left\langle\hat{I}_{k}^{\mathrm{tot}}\right\rangle= & \rho_{S, g g} I_{k, g g}+\rho_{S, e e} I_{k, e e}+\left(Q_{k, g g}-Q_{k, e e}\right) \mathcal{L}_{g g} \\
& +2 \Re e\left(\rho_{S, g e} I_{k, e g}\right)+2 \Re e\left(\mathcal{L}_{g e} Q_{k, e g}\right),
\end{aligned}
$$

where we have used the fact that, from the symmetries of the master equation, $\mathcal{L}_{e e}=-\mathcal{L}_{g g}$ and $\mathcal{L}_{e g}=\mathcal{L}_{g e}^{*}$. We identify the first line as the dynamic current and the second one as the geometric current. Both are composed of standard contributions $I_{k}^{D}=\rho_{S, g g} I_{k, g g}+\rho_{S, e e} I_{k, e e}$ and $I_{k}^{G}=2 \Re e\left(\rho_{S, g e} I_{k, e g}\right)$ and dissipative contributions $I_{k}^{D \text {,diss }}=\left(Q_{k, g g}-Q_{k, e e}\right) \mathcal{L}_{g g}$ and $I_{k}^{G \text {,diss }}=2 \Re e\left(\mathcal{L}_{g e} Q_{k, e g}\right)$. The pumped charge through the $k$ th SQUID is defined as

$$
Q_{k}^{G}=2 \int_{0}^{T_{\mathrm{ad}}} \Re e\left(\rho_{S, g e} I_{k, e g}\right) d t+2 \int_{0}^{T_{\mathrm{ad}}}\left(\mathcal{L}_{g e} Q_{k, e g}\right) d t,
$$

and it is also composed of a standard and a dissipative part.

In the rest of the paper, we consider the current operator for the average current across the device $\hat{I}^{\text {tot }}=\left(\hat{I}_{L}^{\text {tot }}+\hat{I}_{R}^{\text {tot }}\right) / 2$. The standard contributions can be obtained directly from Eq. (10) and explicitly read $\hat{I}_{L}=(2 e / \hbar) J_{L} \sin (\varphi / 2-\hat{\theta})$ and $\hat{I}_{R}=(2 e / \hbar) J_{R} \sin (\varphi / 2+\hat{\theta})$. The dissipative contributions to the current can be calculated using the last term on the righthand side of Eq. (11). However, general expressions similar to the standard contributions do not exist since the dissipative parts depend on the characteristics of the environmental noise and the form of the master equation used. If such details are specified, Eq. (14) allows us to calculate the corresponding average geometric charge $Q^{G}=\left(Q_{L}^{S}+Q_{R}^{S}\right) / 2$, which is the physical observable in our case.

Note that the dissipative contributions $I_{k}^{D \text {,diss }}$ and $I_{k}^{G \text {,diss }}$ are typically small with respect to $I_{k}^{D}$ and $I_{k}^{G}$. If $\lambda$ is the effective coupling between the system and the environment, the dissipative contribution in the master equation $\hat{\mathcal{L}}$ scales as $\lambda^{2}$. Since the master equation, (6), is derived in the limit of weak coupling between the system and the environment, we expect a small contribution in Eq. (13) from the dissipative currents. However, there can be cases in which the dissipative contributions could be detectable if we reduce $I_{k}^{D}$ and $I_{k}^{G}$.

\section{A. Charge noise environment}

When the system is in the charge regime, the main source of noise originates from the fluctuations of the gate voltage. 
References 17-19 focus on the study of this charge noise induced by the environment. If we write the Cooper-pair number operator and the charge on the island as $\hat{n}=\hat{n}_{L}-$ $\hat{n}_{R}$ and $\hat{Q}=-2 e \hat{n}$, respectively, the corresponding systemenvironment interaction is $\hat{H}_{I}=-2 e \lambda \hat{n} \otimes \delta \hat{V}$, where $\lambda$ is the system-environment coupling constant and $\delta \hat{V}$ acts on the environment degrees of freedom. ${ }^{17,18}$ The operators $\hat{n}_{k}$ commute with $\hat{n}$, and from Eq. (10), there is no dissipative current $\left\langle\hat{I}_{k}^{\text {diss }}\right\rangle$ through the $k$ th SQUID and no dissipative contribution to the average current. Therefore, the current is described by the usual definition of the current operator through the SQUIDs, that is, the first term on the right-hand side of Eq. (10), and the corresponding usual current operator across the device. ${ }^{17,18}$ Nevertheless, the transferred charge is influenced by the environment since, as discussed in Sec II, even if the dissipative current vanishes, the total current is modified by the interaction with the environment through the dynamics of $\hat{\rho}_{S}$.

For our result to be consistent, we must check that Eq. (8) is satisfied. Since no dissipative current can be induced by charge noise, i.e., $\left[\hat{Q}, \hat{H}_{I}\right]=0$, Eq. (8) requires that the term $\operatorname{Tr}_{S}(\hat{\mathcal{L}} \hat{Q})$ calculated from the master equation vanishes. This can be verified by a direct calculation using the master equations as discussed in Appendix A. However, this result depends critically on the form of the master equation used and the approximations done. In Refs. 17-19 and 36, the master equation was obtained keeping the nonsecular terms and this procedure produces the expected result. In a similar way, it can be verified that if we use the secular approximation ${ }^{29}$ and perform the same calculation, we obtain $\operatorname{Tr}_{S}\left(\hat{\mathcal{L}}_{\text {sec }} \hat{Q}\right) \neq 0$. This result is in contradiction with the one based simply on a symmetry argument stating that, since $\left[\hat{H}_{I}, \hat{Q}\right]=0$, we do not have a dissipative contribution to the current. This observation is enough to state that the secular approximation is not feasible in the description of Cooper-pair pumping as has already been pointed out earlier. ${ }^{17,18}$ The obtained result is closely related to the general framework of the conservation of operator currents that was introduced in Ref. 33 for essentially any quantum system.

\section{B. Flux noise in a Cooper-pair sluice}

If the Cooper-pair sluice is subject to flux noise, the dissipative contribution to the current is finite and, hence, requires further analysis. As an example, we consider the case in which the fluctuations of the magnetic flux through the outer loop influence the total phase across the sluice $\varphi$. We refer to this as phase bias noise.

We consider small phase fluctuations in the vicinity of the static point $\varphi_{0}$ so that the total phase is $\frac{\varphi}{2}=\frac{\varphi_{0}}{2}+\delta \varphi$, with $\delta \varphi \ll \varphi_{0}$. The total Hamiltonian is $\hat{H}_{J}=\hat{H}_{J}\left(\varphi_{0}\right)+\delta \hat{H}_{J}$, with

$$
\delta \hat{H}_{J}=\left[J_{L} \sin \left(\frac{\varphi_{0}}{2}-\hat{\theta}\right)+J_{R} \sin \left(\frac{\varphi_{0}}{2}+\hat{\theta}\right)\right] \frac{\delta \varphi}{2} .
$$

With the two-state approximation, we can write this in terms of the excess of Cooper pairs on the island $\{|0\rangle,|1\rangle\}$. Using the formulas $e^{i \hat{\theta}}=|1\rangle\langle 0|$ and $e^{-i \hat{\theta}}=|0\rangle\langle 1|$, we have

$$
\delta \hat{H}_{J}=\left(\delta J^{*}|0\rangle\langle 1|+\delta J| 1\rangle\langle 0|\right) \frac{\delta \varphi}{2},
$$

where $\delta J=\left(\sin \frac{\varphi_{0}}{2} J_{+}+i \cos \frac{\varphi_{0}}{2} J_{-}\right)$and $J_{ \pm}=J_{L} \pm J_{R}$.

Equation (16) can be interpreted as the system-environment interaction Hamiltonian when $\delta \varphi$ are induced by the environment. Note that the operator acting on the system degrees of freedom is $\hat{H}_{I, S}=\delta J^{*}|0\rangle\langle 1|+\delta J| 1\rangle\langle 0|$. The charge operator on the island is $\hat{Q}=-2 e|1\rangle\langle 1|$, and since $\left[\hat{H}_{I, S}, \hat{Q}\right] \neq 0$, we must include the contribution of the dissipative current. As discussed above, this contribution can be calculated using the master equation approach. To this end, we can employ the general form of the master equation presented in Ref. 17. The only difference resides in the matrix elements of the coupling operator $\left\langle m\left|\hat{H}_{I, S}\right| n\right\rangle$, where $|n\rangle$ and $|m\rangle$ are the time-dependent eigenstates of the sluice Hamiltonian $\hat{H}_{S}$, and in the spectral density function of the environment. The latter is defined as $S_{\varphi}(\omega)=\int_{-\infty}^{\infty} d \tau\langle\delta \varphi(\tau) \delta \varphi(0)\rangle e^{i \omega \tau}$ and it can be calculated from the correlation function $\langle\delta \varphi(\tau) \delta \varphi(0)\rangle$ of the phase fluctuations.

\section{ENGINEERED ENVIRONMENT FOR PHASE BIAS NOISE}

To determine experimentally and discriminate the effect of environmental noise on Cooper-pair pumping, we should be able to control several properties of the environment. This is possible if, in addition to the natural environment, the system is coupled to an engineered source of noise.

A schematic description of the circuit used for the implementation of such a phase bias environment is presented in Fig. 1. The main source of noise is the thermal resistor $R$ with noise voltage $\delta V$. This circuit is coupled to the system by mutual inductance $M$ and thus it perturbs the phase across the device. To modify the effects of the environment we introduce a control SQUID. By controlling the flux threading it, we can change the current noise in the circuit which is coupled to the system.

The control SQUID can be represented by an $R L C$ parallel circuit with resistance $R_{S}$, inductance $L_{S}$, and capacitance $C_{S}$. The impedance of a single SQUID is $Z_{R L C}=$ $\frac{R_{S} Z_{C_{S}} Z_{L_{S}}}{R_{S} Z_{C_{S}}+Z_{L_{S}} Z_{C_{S}}+R_{S} Z_{L_{S}}}=\frac{i L_{S}(\phi) \omega R_{S}}{i L_{S}(\phi) \omega+R_{S}\left(1-L_{S}(\phi) \omega^{2} C_{S}\right)}$, where $Z_{L_{S}}$ and $Z_{C_{S}}$ are the impedances associated with the inductance $L_{S}$ and capacitance $C_{S}$, respectively. The SQUID inductance $L_{S}(\phi)=L_{0} / \cos \left(\pi \phi / \Phi_{0}\right)$ can be controlled by changing the normalized flux through the SQUID $\phi / \Phi_{0}$. The constant component depends on the maximum critical current of the SQUID $I_{C}: L_{0}=\hbar /\left(2 \pi e I_{C}\right)$. Given $Z_{R L C}$, we can calculate the total impedance of the circuit $Z_{\text {tot }}=Z_{R L C}+Z_{R}+Z_{L}$, where $Z_{R}$ and $Z_{L}$ are the impedances of the resistor and inductor, respectively.

We consider a cold resistor with a cutoff frequency much higher than the typical transition energy of the system $\Omega$, i.e., $\Omega \gg k_{B} T_{R}$ and $\Omega \ll \omega_{c}$, where $\omega_{c}$, and $T_{R}$ are the cutoff frequency and the temperature of the noise source, respectively. In this limit, the spectral density of the JohnsonNyquist voltage noise across the resistor is $S_{V}(\omega)=2 \hbar \omega R$ for $\omega \geqslant 0$ and $S_{V}(\omega)=0$ for $\omega<0$. We obtain the spectral 
density of the current noise $S_{I}(\omega, \phi)=S_{V}(\omega) /\left|Z_{\text {tot }}\right|^{2}$ as

$$
S_{I}(\omega, \phi)=\frac{2 R \hbar \omega}{\left[\frac{\omega^{2} L_{S}(\phi)^{2} R_{S}}{R_{S}^{2}\left(1-C_{S} \omega^{2} L_{S}(\phi)\right)^{2}+\omega^{2} L_{S}(\phi)^{2}}+R\right]^{2}+\omega^{2}\left[\frac{L_{S}(\phi) R_{S}^{2}\left(1-C_{S} \omega^{2} L_{S}(\phi)\right)}{R_{S}^{2}\left(1-C_{S} \omega^{2} L_{S}(\phi)\right)^{2}+L_{S}(\phi)^{2} R_{S}^{2}}+L\right]^{2}} .
$$

The flux noise spectrum is $S_{\Phi}(\omega, \phi)=M^{2} S_{I}(\omega)$, and hence, the phase noise spectrum reads

$$
S_{\varphi}(\omega, \phi)=\frac{4 \pi^{2} M^{2} S_{I}(\omega, \phi)}{\Phi_{0}^{2}} .
$$

The behavior of $S_{\varphi}(\omega, \phi)$ as a function of the SQUID flux $\phi$ and for a fixed frequency $\omega_{m}$ is shown in Fig. 2(a). The spectrum shows two resonances at $\phi / \Phi_{0}=1 / 2,3 / 2$ and it reflects the behavior of the control SQUID inductance $L_{S}(\phi)$. At these points the artificial environment is maximally decoupled.

It is useful to analyze in detail the behavior of the spectrum near the resonance $\phi / \Phi_{0}=1 / 2$. The spectral density function in Eq. (18) for realistic circuit parameters used in the numerical simulations (see Fig. 2) is well approximated by

$$
S_{\varphi}\left(\omega_{m}, \phi\right)=\frac{8 \pi^{2} M^{2} R \hbar \omega_{m}}{\Phi_{0}^{2}\left\{R^{2}+L^{2} \omega_{m}^{2}\left[1-\frac{L_{0}}{\pi L\left(\phi / \Phi_{0}-1 / 2\right)}\right]^{2}\right\}} .
$$

This expression allows us to calculate the positions and the values of the maximum and the minimum of the spectral density function. The maximum is found at $\phi_{\max } / \Phi_{0}=$ $1 / 2+L_{0} /(\pi L)$ and it is $S_{\varphi}^{\max }\left(\omega_{m}, \phi_{\max }\right)=\frac{8 \pi^{2} M^{2} \hbar \omega_{m}}{R \Phi_{0}^{2}}$. The minimum can be obtained from Eq. (17) in the limit $\phi / \Phi_{0} \rightarrow 1 / 2$ and it is denoted $S_{\varphi}^{\min }\left(\omega_{m}, 1 / 2\right)$. The width of the drop is given by the difference $\phi_{\max } / \Phi_{0}-1 / 2=$ $L_{0} /(\pi L)$. It is determined by the ratio between the minimum inductance of the SQUID and the inductance of the circuit [see inset in Fig. 2(a)]. Using the circuit parameters in Fig. 2, we can estimate the decrease in the noise spectrum at the minimum: $\left.S_{\varphi}^{\min }\left(\omega_{m}, 1 / 2\right) / S_{\varphi}^{\max }\left(\omega_{m}, \phi_{\max }\right)\right\} \approx 10^{-4}$ and $S_{\varphi}^{\min }\left(\omega_{m}, 1 / 2\right) / S_{\varphi}\left(\omega_{m}, 0\right) \approx 10^{-3}$. This means that we can reduce the strength of the system-environment interaction and effectively decouple the system from the artificial noise source.

The noise influences the circuit at a frequency equal to the energy gap $\Omega$ in the system. In steered evolution $\Omega$ changes in time and so does the coupling frequency. To have an estimate of this effect, Fig. 2(b) shows the change in the spectrum as the ratio $S_{\varphi}(\omega, \phi) / S_{\varphi}(\omega, 0)$ for the range of frequencies $\Omega$ spanned in the evolution during the cycle in Fig. 1(b). Here controlling the SQUID drastically reduces the strength and spectral density of the noise during evolution.

We note that the corresponding decoherence processes induced by artificial noise are stronger than those naturally produced by the flux noise typically observed in experiments (see Appendix B). For this reason, we neglect the effects of the latter and consider only the decoherence induced by the engineered environment.

In the following we also neglect charge noise. An estimate of the artificial flux noise strength (see Appendix B) shows that, on average, it is stronger than the charge noise except that near the resonance points of $S_{\varphi}(\omega, \phi)$. Thus, the effect of artificial flux noise near these regions would be partially hidden. To observe clearly the influence of flux noise for all values of $\phi$, it is necessary to reduce the charge noise or to further increase the strength of the flux noise coupling, i.e., increase the mutual inductance.

\section{NUMERICAL RESULTS}

Let us study the effect of the artificial noise on the pumped charge in the device shown in Fig. 1(a). The SQUID energies and the gate voltage are modulated within the adiabatic time $T_{\mathrm{ad}}=1 / f$, where $f$ is the pumping frequency. The system is initialized in the ground state and the dynamics is obtained by
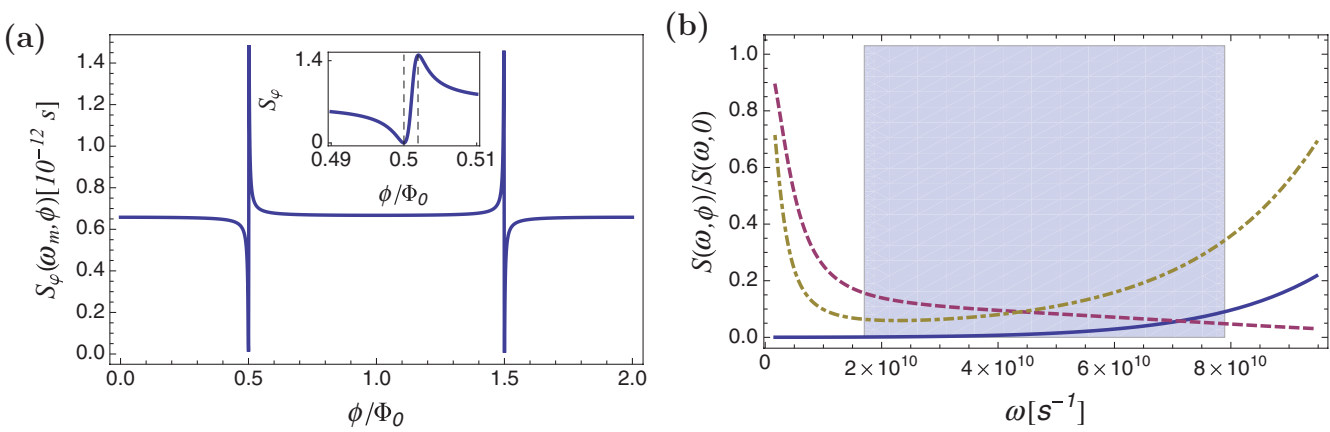

FIG. 2. (Color online) (a) Spectral density function of the phase bias noise as a function of the magnetic flux through the control SQUID $\phi / \Phi_{0}$ for frequency $\omega_{m}=1.7 \times 10^{10} \mathrm{~s}^{-1}$. Inset: A zoom near the resonance point $\phi / \Phi_{0}=1 / 2$. Dashed lines show the region delimited by the minimum and maximum $S_{\varphi}\left(\omega_{m}, \phi\right)$. Its width is given by $L_{0} /(\pi L)$. (b) The ratio $S_{\varphi}(\omega, \phi) / S_{\varphi}(\omega, 0)$ for $\phi / \Phi_{0}=0.5$ (solid line), $\phi / \Phi_{0}=0.4991$ (dashed line), and $\phi / \Phi_{0}=0.5003$ (dot-dashed line). The shaded region denotes the frequency range spanned during the typical pumping cycle shown in Fig. 1(b): $1.7 \times 10^{10} \mathrm{~s}^{-1} \leqslant \omega \leqslant 7.9 \times 10^{10} \mathrm{~s}^{-1}$. The parameters of the artificial noise are $R=30 \Omega, R_{S}=500 \Omega, C_{S}=50 \mathrm{fF}$, $I_{C}=25 \mu \mathrm{A}$, and $L=M=0.69 \mathrm{nH}$. 

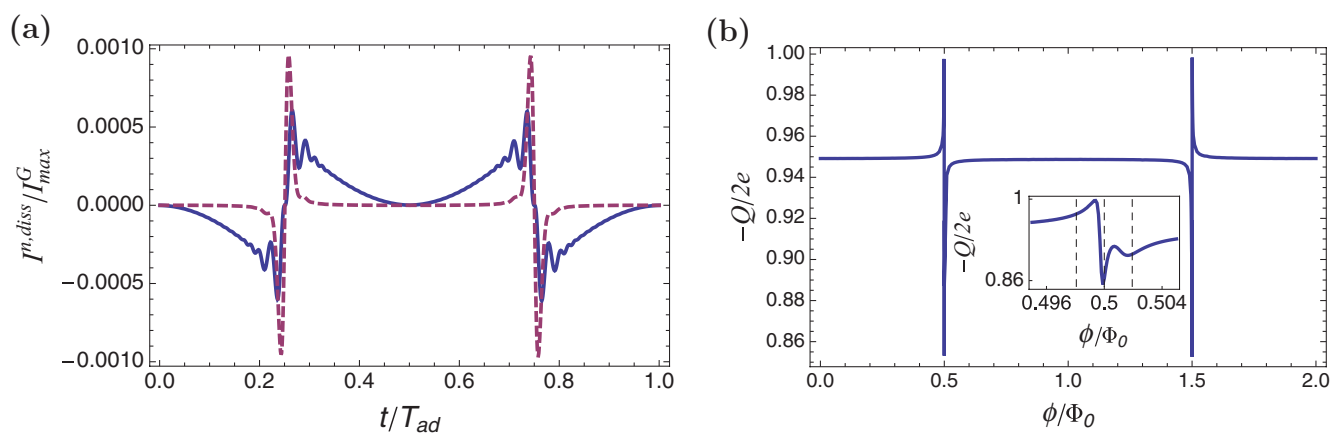

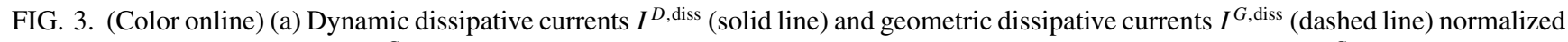
to the maximum geometric current $I_{\max }^{G}$ during the pumping cycle. The maximum geometric current during one cycle is $I_{\max }^{G}=0.5 \mathrm{nA}$ and the control SQUID of the environmental circuit is open with $\phi / \Phi_{0}=1$. (b) Pumped charge as a function of the flux through the control SQUID of the artificial environment circuit. Inset: Behavior near one of the resonance points. Dashed lines are determined from Fig. 2(a) and they are located at the minimum $\phi / \Phi_{0}=1 / 2$ and at $\phi / \Phi_{0}=1 / 2 \pm L_{0} /(\pi L)$ of the spectral density function. In numerical simulations, the phase bias is $\varphi_{0}=\pi / 2$, the pumping frequency is $f=150 \mathrm{MHz}$, and the device parameters are $J_{i}^{M} / E_{\mathrm{C}}=0.1, J_{i}^{m} / J_{i}^{M}=0.03$ (with $i=L, R$ ), $n_{g}^{M}=0.8$ $n_{g}^{m}=0.2$, and $E_{\mathrm{C}} / k_{\mathrm{B}}=1 \mathrm{~K}\left[E_{\mathrm{C}} /(2 \pi \hbar)=21 \mathrm{GHz}\right]$.

solving the master equation for the reduced density operator up to the first order in the adiabatic parameter. ${ }^{17,18}$ The pumping cycle is repeated until the system reaches the steady-state solution $\rho_{S}\left(t+T_{\mathrm{ad}}\right)=\rho_{S}(t)$. We assume that the dissipative source $R$ is at such a low temperature that the environment cannot excite the system.

In Fig. 3(a), we show the time dependence of the dissipative dynamic and geometric currents induced by the phase bias noise. Curves correspond to dissipative currents flowing through the circuit when the system reaches the steadystate solution. The dissipative currents are about 3 orders of magnitude smaller than the total geometric current. The corresponding dissipative pumped charge is almost 0 . This is not surprising since we are analyzing the system in the steady-state solution, and since the dissipative currents have no favored direction, the corresponding charge averages out over many pumping cycles. The dissipative contributions are significantly greater during the first pumping cycles when the system is still far from steady state.

In Fig. 3(b), we present the stationary pumped charge under the influence of artificial flux noise. The tuning parameter is the flux of the control SQUID $\phi / \Phi_{0}$, which allows us to effectively change the system-environment coupling. As shown, the pumped charge is almost constant except near the resonance points $\phi / \Phi_{0}=1 / 2,3 / 2$. The global behavior of the pumped charge and its drop at the resonance points can be physically explained keeping in mind that here the systemenvironment coupling is effectively suppressed [Fig. 2(a)]. The stationary pumped charge is determined by two competing effects. Nonadiabatic transitions tend to excite the system, while the relaxation induced by the environment tends to keep the system in the ground state. Since the ground and excited states pump in opposite directions, the first effect reduces the pumped charge while the second one stabilizes it near the expected value of one Cooper pair per cycle. ${ }^{17,18,43}$ The stationary solution is reached after many pumping cycles when the two effects are in balance. Away from the resonance points, the spectrum is almost constant, producing a constant pumped charge. At the resonances, the results in Fig. 3(b) reflect the fact that, since we are effectively decreasing the system-environment coupling, nonadiabatic excitations become more important. Correspondingly, the excited-state component is enhanced in the final steady state and we observe a smaller pumped charge. The maximum drop of the pumped charge is $\left(Q_{\max }-Q_{\min }\right) / Q_{\max } \approx 15 \%$ and the drop from the pumped charge away from the resonance $\left(Q_{0}-Q_{\min }\right) / Q_{0} \approx$ $10 \%\left[Q_{0}=Q(\phi=0)\right]$.

However, the details of the pumped current in the proximity of resonances are different from those exhibited by the environment spectrum $S_{\varphi}(\omega, \phi)$. This reflects the fact that the pumped charge is an observable that can be influenced by many effects. In particular, the pumped charge first increases near the resonance points, before decreasing. It has clearly nontrivial behavior [inset in Fig. 3(b)]. One possible explanation of this behavior is the influence of Landau-Zener-Stückelberg (LZS) interference. ${ }^{39}$ During the pumping cycle in Fig. 1(b), the system crosses two avoided crossings where $n_{g}=1 / 2$. Near these points Landau-Zener transitions occur and the phase difference between the excited and the ground state accumulated in the intermediate region leads to interference effects. The overall result is a change in the ground-state population and thus the pumped charge. In the present model, the phase bias is kept constant but artificial noise can effectively induce a change in the accumulated phase difference and hence in the LZS interference. If the system is initially in the ground state, the probability of exciting it due to LZS interferometry after one cycle is $\mathcal{P}_{e}=P_{\mathrm{LZ}}\left(1-P_{\mathrm{LZ}}\right) \cos ^{2}(\alpha+\varphi / 2)$, where $P_{L Z}$ is the Landau-Zener transition probability and $\alpha$ depends on the energy gap and on the pumping frequency. ${ }^{39}$ If $\delta \varphi$ is the perturbation on the constant phase bias $\varphi_{0}=\pi / 2$, we have $\varphi / 2=\pi / 4+\delta \varphi$. The average of the excitation probability during a loop is

$$
\mathcal{P}_{e} \approx \mathcal{P}_{e}^{0}+\frac{1}{2} P_{\mathrm{LZ}}\left(1-P_{\mathrm{LZ}}\right) \sin (2 \alpha)\left\langle\delta \varphi^{2}\right\rangle,
$$

where $\mathcal{P}_{e}^{0}$ is the excitation probability without noise, $\left\langle\delta \varphi^{2}\right\rangle$ is the average of the square phase fluctuation, and we have assumed $\langle\delta \varphi\rangle=0$. We calculated the contribution due to the noise as $\left\langle\delta \varphi^{2}\right\rangle=\int_{\omega_{m}}^{\omega_{M}} S_{\varphi}(\omega, \phi) d \omega$, and as a function of the control flux $\phi$, and observe that it behaves as $S_{\varphi}(\omega, \phi)$ as a 
function of $\phi$. The probability of remaining in the ground state is $\mathcal{P}_{g}=1-\mathcal{P}_{e}$ and its dependence on the control SQUID flux $\phi$ is inverted with respect to $S(\omega, \phi)$. This means that when the strength of the noise spectrum increases because of the LZS effect, the ground state is less populated and the pumped charge decreases. The final behavior of the pumped charge as a function of $\phi$ is determined by the noise spectrum, the LZS interference, and their relative strength integrated over the pumping cycles.

Note that for numerical simulations we have used the master equation which includes only the first-order contribution in the perturbative adiabatic parameter. ${ }^{17,18}$ To obtain a more accurate value of the pumped charge a different master equation which includes high-order corrections can be used. ${ }^{19,36}$

\section{CONCLUSIONS}

In summary, we have presented a study of flux noise on the Cooper-pair pumping process. First, we showed that in the presence of flux noise the current operator must be modified in order to take into account the current induced by the environment. This dissipative current is related to the symmetry of the system and can be calculated from the master equation for the reduced density matrix.

Second, we analyzed the effects of phase bias noise on Cooper-pair pumping. In the model studied, the noise is produced by an artificial environment coupled to the system by mutual inductance. The advantage of this scheme is that, introducing a control SQUID in the environmental circuit, we have access to the system-environment coupling and can essentially decouple the system from the environment. The pumped charge obtained by solving the master equation clearly shows the features induced by noise.

The system presented here operates in the charge regime and it is then primarily sensitive to charge noise. For this reason, it can be challenging to experimentally measure the predicted effect because it can be partially hidden by the charge noise. However, there are several modifications which can increase the experimental accessibility: reducing the effect of charge noise and increasing the mutual inductance coupling with a different design of the artificial noise circuit. For example, using multilayer lithographic techniques, it is possible to increase the coupling between the system and the environment.

This work is a step toward understanding the effect of different types of noises on steered superconducting devices. Such understanding is critical for practical applications such as metrology and quantum information processing. A deeper knowledge of the effect of the environment can open a way to the design and implementation of robust devices or, in the case of quantum information, error correction techniques.

In this direction, the pioneering experimental works of implementing engineered environments have been carried out using trapped ions. ${ }^{44,45}$ One of the most striking results of these experiments was the proof that it is possible to store quantum information in states which are robust against decoherence. ${ }^{45}$ Still, apart from a few theoretical proposals, ${ }^{18}$ similar experiments are missing in condensed matter systems.

Along these lines, the next step is to analyze the effect of similar flux noise sources on superconducting devices in different regimes. For example, in the transmon regime the system is insensitive to charge noise, and flux noise becomes the dominant source of noise. ${ }^{46}$ Thus, using the discussed engineered environment, we should be able to study and measure the environmental effect in controlled situations.

\section{ACKNOWLEDGMENTS}

We received funding from the European Community's Seventh Framework Programme under Grant Agreement No. 238345 (GEOMDISS). We acknowledge the Academy of Finland, Emil Aaltonen Foundation, Väisälä Foundation, and KAUTE Foundation for financial support.

\section{APPENDIX A: ABSENCE OF DISSIPATIVE CURRENT WITH A CHARGE NOISE ENVIRONMENT}

By using Eq. (8) we can check by a direct calculation that, when the system is subjected to charge noise, there is no dissipative current contribution. When the system is adiabatically steered along a cyclic loop, the master equation is conveniently written in the time-dependent eigenbasis of $\hat{H}_{S}$. There are several equivalent approaches ${ }^{17-19,36}$ exploiting the two-level approximation in the weak coupling limit. Since the present calculation can be done in an abstract way without explicitly taking into consideration the form of $\hat{H}_{S}$, it is convenient to use the master equation in the superadiabatic basis representation, ${ }^{19,36}$ which is easier to manipulate.

Let $\hat{D}_{1}$ be the operator which diagonalizes $\hat{H}_{S}$ in a fixed basis. Then the dynamics of the closed system are effectively governed by $\hat{H}_{S, 1}=\hat{D}_{1}^{\dagger} \hat{H}_{S} \hat{D}_{1}+\hbar \hat{w}_{1}$, where $\hat{w}_{1}=-i \hat{D}_{1}^{\dagger} \dot{\hat{D}}_{1}$. Defining $\hat{D}_{2}$ as the diagonalizing operator for $\hat{H}_{S, 1}$ allows us to define a new effective Hamiltonian as $\hat{H}_{S, 2}=\hat{D}_{2}^{\dagger} \hat{H}_{S} \hat{D}_{2}+$ $\hbar \hat{w}_{2}$, where $\hat{w}_{2}=-i \hat{D}_{2}^{\dagger} \dot{\hat{D}}_{2}$. If the evolution is sufficiently slow, that is, $\left|\hat{w}_{2}\right| \ll\left|\hat{w}_{1}\right|$, we may approximate $\hat{H}_{S, 2} \approx$ $\hat{D}_{2}^{\dagger} \hat{H}_{S} \hat{D}_{2}$, yielding an effectively nonsteered picture. Denoting the superadiabatic basis $\left\{|g\rangle=\hat{D}_{1} \hat{D}_{2}|0\rangle,|e\rangle=\hat{D}_{1} \hat{D}_{2}|1\rangle\right\}$, where $\{|0\rangle,|1\rangle\}$ defines the fixed basis, yields a master equation in the Schrödinger picture as

$$
\frac{d \rho_{g g}}{d t}=-\left(\Gamma_{g e}+\Gamma_{e g}\right) \rho_{g g}+\Re e\left\{\tilde{\Gamma}_{0} \rho_{g e}\right\}+\Gamma_{e g},
$$

and

$$
\begin{aligned}
\frac{d \rho_{g e}}{d t}= & i \Omega \rho_{g e}-\left(\tilde{\Gamma}_{+}+\tilde{\Gamma}_{-}\right) \rho_{g g}\left(\frac{\Gamma_{e g}}{2}+\frac{\Gamma_{g e}}{2}+\Gamma_{\varphi}\right) \rho_{g e} \\
& +\left(\Gamma_{\alpha}+\Gamma_{\beta}\right) \rho_{e g}+\tilde{\Gamma}_{+},
\end{aligned}
$$

where $\rho_{r s}=\left\langle r\left|\hat{\rho}_{S}\right| s\right\rangle$ with $r, s \in\{g, e\}$, and $\Omega=\left(E_{e}-E_{g}\right) / \hbar$. If $\hat{H}_{I}=\hat{Z} \otimes \hat{X}$, where $\hat{Z}$ and $\hat{X}$ are the operators acting on the system and the environment, respectively, the rates in Eqs. (A1) and (A2) are defined as

$$
\begin{aligned}
\Gamma_{g e} & =\frac{|\langle e|\hat{Z}| g\rangle|^{2}}{\hbar^{2}} S(-\Omega), \\
\Gamma_{e g} & =\frac{|\langle e|\hat{Z}| g\rangle|^{2}}{\hbar^{2}} S(+\Omega), \\
\tilde{\Gamma}_{0} & =\frac{\langle e|\hat{Z}| g\rangle(\langle g|\hat{Z}| g\rangle-\langle e|\hat{Z}| e\rangle)}{\hbar^{2}} S(0),
\end{aligned}
$$




$$
\begin{aligned}
\tilde{\Gamma}_{ \pm} & =\frac{\langle g|\hat{Z}| e\rangle(\langle e|\hat{Z}| e\rangle-\langle g|\hat{Z}| g\rangle)}{2 \hbar^{2}} S( \pm \Omega), \\
\Gamma_{\varphi} & =\left(\frac{|\langle e|\hat{Z}| e\rangle|^{2}}{2 \hbar^{2}}+\frac{|\langle g|\hat{Z}| g\rangle|^{2}}{2 \hbar^{2}}-\frac{\langle g|\hat{Z}| g\rangle\langle e|\hat{Z}| e\rangle}{\hbar^{2}}\right) S(0), \\
\Gamma_{\alpha} & =\frac{\langle g|\hat{Z}| e\rangle^{2}}{2 \hbar^{2}} S(\Omega), \\
\Gamma_{\beta} & =\frac{\langle g|\hat{Z}| e\rangle^{2}}{2 \hbar^{2}} S(-\Omega) .
\end{aligned}
$$

The spectral density is denoted $S(\omega)=\int_{-\infty}^{\infty} d \tau\langle\hat{X}(\tau)$ $\hat{X}(0)\rangle e^{i \omega \tau}$. Note that Eqs. (A1) and (A2) still contain the nonsecular terms.

We can write the superadiabatic states in a general form as

$$
\begin{aligned}
& |g\rangle=\cos \theta|0\rangle+\sin \theta e^{i \phi}|1\rangle, \\
& |e\rangle=\sin \theta|0\rangle-\cos \theta e^{-i \phi}|1\rangle,
\end{aligned}
$$

where $\theta$ and $\gamma$ are time-dependent functions. We consider a generic diagonal noise operator in the charge basis, $\hat{Z}=$ $a|1\rangle\langle 1|+b| 0\rangle\langle 0|$ for $a, b \in \mathbb{R}$. Expressing the charge operator of the island as $\hat{Q}=-2 e|1\rangle\langle 1|$ and the noise operator $\hat{Z}$ in the time-dependent basis (A4), we have

$$
\begin{aligned}
\hat{Q}= & -2 e\left[\sin ^{2} \theta|g\rangle\left\langle g\left|+\cos ^{2} \theta\right| e\right\rangle\langle e|\right. \\
& -\cos \theta \sin \theta(|e\rangle\langle g|+| g\rangle\langle e|)]
\end{aligned}
$$

and

$$
\begin{aligned}
\hat{Z}= & \left(a \cos ^{2} \theta+b \sin ^{2} \theta\right)|g\rangle\langle g| \\
& +\left(b \cos ^{2} \theta+a \sin ^{2} \theta\right)|e\rangle\langle e| \\
& \times(a-b) \cos \theta \sin \theta(|e\rangle\langle g|+| g\rangle\langle e|) .
\end{aligned}
$$

At the same time the $\mathcal{L}_{i j}$ terms in Eq. (7) can be read directly from the dissipative part of the master equation,

$$
\begin{aligned}
\mathcal{L}_{g g}= & -\left(\Gamma_{g e}+\Gamma_{e g}\right) \rho_{g g}+\Re e\left\{\tilde{\Gamma}_{0} \rho_{g e}\right\}+\Gamma_{e g}, \\
\mathcal{L}_{g e}= & -\left(\tilde{\Gamma}_{+}+\tilde{\Gamma}_{-}\right) \rho_{g g}-\left(\frac{\Gamma_{e g}}{2}+\frac{\Gamma_{g e}}{2}+\Gamma_{\varphi}\right) \rho_{g e} \\
& +\left(\Gamma_{\alpha}+\Gamma_{\beta}\right) \rho_{e g}+\tilde{\Gamma}_{+} .
\end{aligned}
$$

Thus we have all the elements to calculate the dissipative current

$$
\operatorname{Tr}_{S}(\hat{\mathcal{L}} \hat{Q})=\left(Q_{g g}-Q_{e e}\right) \mathcal{L}_{g g}+2 \Re e\left(\mathcal{L}_{g e} Q_{e g}\right) .
$$

By explicitly writing the transition rate in Eq. (A3) for the noise operator $\hat{Z}$ and inserting all the contributions in Eq. (A8), we verify that $\operatorname{Tr}_{S}(\hat{\mathcal{L}} \hat{Q})=0$. These results are valid for any perturbation operator diagonal in the $\{|0\rangle,|1\rangle\}$ basis and thus for the charge noise discussed in Sec. III A. The same result can be obtained using the master equation in the adiabatic basis. ${ }^{17,18}$

It is interesting that the condition $\operatorname{Tr}_{S}(\hat{\mathcal{L}} \hat{Q})=0$ depends critically on the form of the master equation. As an example, we consider the same problem, but we perform the usual secular approximation. ${ }^{29}$ The correctness of the secular approximation in the analysis of the charge pumping has been questioned $^{17,18}$ since it has been shown that it can lead to unphysical results such as charge nonconservation. The master equation after the secular approximation reads

$$
\frac{d \rho_{g g}^{\mathrm{sec}}}{d t}=-\left(\Gamma_{g e}+\Gamma_{e g}\right) \rho_{g g}^{\mathrm{sec}}+\Gamma_{e g}
$$

and

$$
\frac{d \rho_{g e}^{\mathrm{sec}}}{d t}=i \Omega \rho_{g e}^{\mathrm{sec}}-\left(\frac{\Gamma_{e g}}{2}+\frac{\Gamma_{g e}}{2}+\Gamma_{\varphi}\right) \rho_{g e}^{\mathrm{sec}} .
$$

In this case, it can be shown that a calculation similar to the one above gives nonvanishing dissipative current since $\operatorname{Tr}_{S}\left(\hat{\mathcal{L}}_{\mathrm{sec}} \hat{Q}\right) \neq 0$. This result is in contradiction to the result based on the symmetry argument. Similar results can be obtained in a more formal framework as discussed in Ref. 33.

\section{APPENDIX B: ARTIFICIAL AND NATURAL DECOHERENCE RATES}

In addition to the artificial environment discussed in Sec. IV, we should also take into account the effect of natural flux noise. It is sufficient to consider the generic decoherece rate $\Gamma(\phi)=|\langle i|\delta \hat{H}| j\rangle / \hbar|^{2} S_{\varphi}(\omega, \phi)$, where $S_{\varphi}(\omega, \phi)$ is the spectral density function of the environment. The matrix element $|\langle i|\delta \hat{H}| j\rangle|^{2}$ is associated with the relaxation process if $i \neq j$ and with the dephasing if $i=j$. The order of magnitude of $|\langle i|\delta \hat{H}| j\rangle / \hbar|^{2}$ for the pumping cycle in Fig. 1(b) can be estimated using Eq. (16) as $\left(J_{M} / \hbar\right)^{2}$. Combining this with the values of $S_{\varphi}(\omega, \phi)$, we have an estimate of the decoherence times (both dephasing and relaxation), $\tau(\phi)=1 / \Gamma(\phi)$.

During the pumping cycle the system energy gap changes in time $e^{17,18}$ and the minimum energy gap is reached near the degeneracy points where $n_{g}=1 / 2$. For the majority of the evolution time, the system frequency is close to its maximum $\omega_{M}=7.9 \times 10^{10} \mathrm{~s}^{-1}$. We can use this reference frequency to estimate the decoherence rates. The minimum and maximum values of the spectral density function at $\omega_{M}$ are $S_{\varphi}^{\min }=2.8 \times$ $10^{-15} \mathrm{~s}$ and $S_{\varphi}^{\max }=3 \times 10^{-12} \mathrm{~s}$. The estimated relaxation and dephasing times with the parameters used in the numerical simulation (see caption to Fig. 3 ) are $\tau_{\min } \approx 1.9$ ns and $\tau_{\max } \approx$ $2 \mu \mathrm{s}$.

For charge noise, the decay and dephasing times can be estimated as in Ref. 17 and they are of the order $10 \mathrm{~ns}$. Thus, artificial flux noise is the dominant source of noise except that near the resonance points of $S_{\varphi}(\omega, \phi)$.

The effect of the natural environment can be estimated from the experimental results of the decoherence time for devices affected by flux. ${ }^{47-49}$ We focus on the effect of ubiquitous $1 / f$ noise. In this case, measurements of the decoherence induced by such noise suggest that the decay has a Gaussian and not an exponential shape. ${ }^{48,49}$ Note that, strictly speaking, this implies that it cannot be described by a linear master equation. However, a phenomenological approach has been applied. ${ }^{47}$ The effective dephasing rate is $\Gamma=\sqrt{A_{\varphi} \ln 2}|\partial \Omega / \partial \varphi|$, where the spectral density function for $1 / f$ noise is $S_{\varphi}(\omega)=A_{\varphi} /|\omega|$ and $\Omega$ is the frequency of the system. A maximum of $|\partial \Omega / \partial \varphi|$ is obtained when the system is at the degeneracy point, i.e., $n_{g}=1 / 2$ and only the Josephson contribution is present in the Hamiltonian [see Eq. (9)]. In this case, 
$\Omega=\sqrt{J_{R}^{2}+J_{L}^{2}+2 J_{R} J_{L} \cos \varphi} / \hbar$ and we can approximate $J_{R} \approx J_{M} \gg J_{L} \approx J_{m}$. From the measured amplitude of the magnetic flux spectrum $A_{\Phi}=\left(1.7 \mu \Phi_{0}\right)^{2}$, we can calculate the amplitude of the phase spectrum $A_{\varphi}=4 \pi^{2} A_{\Phi} / \Phi_{0}^{2}$. Thus, we obtain the dephasing time due to low-frequency noise: $\tau \approx 28 \mu \mathrm{s}$. This dephasing time is very long compared to the artificial decoherence time, and hence the natural lowfrequency noise does not change the results of the numerical simulations presented . *solpaolo@gmail.com

${ }^{1}$ M. V. Berry, Proc. R. Soc. London A 392, 45 (1984).

${ }^{2}$ F. Wilczek and A. Zee, Phys. Rev. Lett. 52, 2111 (1984).

${ }^{3}$ G. Falci, R. Fazio, G. M. Palma, J. Siewert, and V. Vedral, Nature (London) 407, 355 (2000).

${ }^{4}$ P. Zanardi and M. Rasetti, Phys. Lett. A 264, 94 (1999).

${ }^{5}$ G. De Chiara and G. M. Palma, Phys. Rev. Lett. 91, 090404 (2003).

${ }^{6}$ P. Solinas, P. Zanardi, and N. Zanghí, Phys. Rev. A 70, 042316 (2004).

${ }^{7}$ See, e.g., A. Tomita and R. Y. Chiao, Phys. Rev. Lett. 57, 937 (1986).

${ }^{8}$ P. J. Leek et al., Science 318, 1889 (2007).

${ }^{9}$ M. Möttönen, J. J. Vartiainen, and J. P. Pekola, Phys. Rev. Lett. 100, 177201 (2008).

${ }^{10}$ R. G. Unanyan, B. W. Shore, and K. Bergmann, Phys. Rev. A 59, 2910 (1999).

${ }^{11}$ L.-M. Duan, J. I. Cirac, and P. Zoller, Science 292, 1695 (2001).

${ }^{12}$ I. Fuentes-Guridi, J. Pachos, S. Bose, V. Vedral, and S. Choi, Phys. Rev. A 66, 022102 (2002).

${ }^{13}$ A. Recati, T. Calarco, P. Zanardi, J. I. Cirac, and P. Zoller, Phys. Rev. A 66, 032309 (2002).

${ }^{14}$ L. Faoro, J. Siewert, and R. Fazio, Phys. Rev. Lett. 90, 028301 (2003).

${ }^{15}$ P. Solinas, P. Zanardi, N. Zanghì, and F. Rossi, Phys. Rev. B 67, 121307(R) (2003).

${ }^{16}$ P. Zhang, Z. D. Wang, J. D. Sun, and C. P. Sun, Phys. Rev. A 71, 042301 (2005).

${ }^{17}$ J. P. Pekola, V. Brosco, M. Möttönen, P. Solinas, and A. Shnirman, Phys. Rev. Lett. 105, 030401 (2010).

${ }^{18}$ P. Solinas, M. Möttönen, J. Salmilehto, and J. P. Pekola, Phys. Rev. B 82, 134517 (2010).

${ }^{19}$ J. Salmilehto and M. Möttönen, Phys. Rev. B 84, 174507 (2011).

${ }^{20}$ A. O. Niskanen, J. P. Pekola, and H. Seppä, Phys. Rev. Lett. 91, 177003 (2003).

${ }^{21}$ M. Aunola and J. J. Toppari, Phys. Rev. B 68, 020502(R) (2003).

${ }^{22}$ M. Möttönen, J. P. Pekola, J. J. Vartiainen, V. Brosco, and F. W. J. Hekking, Phys. Rev. B 73, 214523 (2006).

${ }^{23}$ V. Brosco, R. Fazio, F. W. J. Hekking, and A. Joye, Phys. Rev. Lett. 100, 027002 (2008).

${ }^{24}$ J.-M. Pirkkalainen, P. Solinas, J. P. Pekola, and M. Möttönen, Phys. Rev. B 81, 174506 (2010).

${ }^{25}$ P. Solinas, J.-M. Pirkkalainen, and M. Möttönen, Phys. Rev. A 82, 052304 (2010).
${ }^{26}$ I. Kamleitner, P. Solinas, C. Müller, A. Shnirman, and M. Möttönen, Phys. Rev. B 83, 214518 (2011).

${ }^{27}$ M. Neeley et al., Science 325, 722 (2009).

${ }^{28}$ M. Pechal, S. Berger, A. A. Abdumalikov Jr., A. Wallraff, and S. Filipp, e-print arXiv:1109.1157v1.

${ }^{29} \mathrm{~K}$. Blum, Density Matrix Theory and Applications (Plenum Press, New York, 1996).

${ }^{30}$ R. Gebauer and R. Car, Phys. Rev. Lett. 93, 160404 (2004).

${ }^{31}$ A. Bodor and L. Diósi, Phys. Rev. A 73, 064101 (2006).

${ }^{32}$ J. J. Prachař and T. Novotný, Physica E 42, 565 (2010).

${ }^{33}$ J. Salmilehto, P. Solinas, and M. Möttönen, e-print arXiv: 1110.5427.

${ }^{34}$ A. O. Niskanen, J. M. Kivioja, H. Seppä, and J. P. Pekola, Phys. Rev. B 71, 012513 (2005).

${ }^{35}$ J. J. Vartiainen, M. Möttönen, and J. P. Pekola, Appl. Phys. Lett. 90, 082102 (2007).

${ }^{36}$ J. Salmilehto, P. Solinas, J. Ankerhold, and M. Möttönen, Phys. Rev. A 82, 062112 (2010).

${ }^{37}$ A. Russomanno, S. Pugnetti, V. Brosco, and R. Fazio, Phys. Rev. B 83, 214508 (2011).

${ }^{38}$ I. Kamleitner and A. Shnirman, Phys. Rev. B 84, 235140 (2011).

${ }^{39}$ S. Gasparinetti, P. Solinas, and J. P. Pekola, Phys. Rev. Lett. 107, 207002 (2011)

${ }^{40}$ R. Leone, L. P. Levy, and P. Lafarge, Phys. Rev. Lett. 100, 117001 (2008).

${ }^{41}$ R. Leone and L. Levy, Phys. Rev. B 77, 064524 (2008).

${ }^{42} \mathrm{~A}$ similar Hamiltonian with a nontunable energy junction was derived for the Bloch transistor by D. V. Averin and K. K. Likharev, in Mesoscopic Phenomena in Solids, edited by B. L. Altshuler et al. (Elsevier, Amsterdam, 1991).

${ }^{43}$ We recall that we are using a master equation up to the first order in the adiabatic parameter. A more accurate prediction for the pumped charge when the system-environment coupling increases can be obtained including higher order corrections.

${ }^{44}$ C. J. Myatt, B. E. King, Q. A. Turchette, C. A. Sackett, D. Kielpinski, W. M. Itano, C. Monroe, and D. J. Wineland, Nature 403, 269 (2000).

${ }^{45}$ D. Kielpinski, V. Meyer, M. A. Rowe, C. A. Sackett, W. M. Itano, C. Monroe, and D. J. Wineland, Science 291, 1013 (2001).

${ }^{46}$ J. Koch, T. M. Yu, J. Gambetta, A. A. Houck, D. I. Schuster, J. Majer, A. Blais, M. H. Devoret, S. M. Girvin, and R. J. Schoelkopf, Phys. Rev. A 76, 042319 (2007).

${ }^{47}$ G. Ithier et al., Phys. Rev. B 72, 134519 (2005).

${ }^{48}$ F. Yoshihara, K. Harrabi, A. O. Niskanen, Y. Nakamura, and J. S. Tsai, Phys. Rev. Lett. 97, 167001 (2006).

${ }^{49}$ J. Bylander et al., Nature Phys. 7, 565 (2011). 